\title{
An optimal iterative algorithm to solve Cauchy problem for Laplace equation
}

\author{
Muhammad Usman Majeed and Taous Meriem Laleg-Kirati ${ }^{\star}$
}

\begin{abstract}
An optimal mean square error minimizer algorithm is developed to solve severely ill-posed Cauchy problem for Laplace equation on an annulus domain. The mathematical problem is presented as a first order state space-like system and an optimal iterative algorithm is developed that minimizes the mean square error in states. Finite difference discretization schemes are used to discretize first order system. After numerical discretization algorithm equations are derived taking inspiration from Kalman filter however using one of the space variables as a time-like variable. Given Dirichlet and Neumann boundary conditions are used on the Cauchy data boundary and fictitious points are introduced on the unknown solution boundary. The algorithm is run for a number of iterations using the solution of previous iteration as a guess for the next one. The method developed happens to be highly robust to noise in Cauchy data and numerically efficient results are illustrated.
\end{abstract}

Keywords-Optimal iterative algorithm, Cauchy problem, Laplace equation, inverse problems, robust to noise.

\section{INTRODUCTION}

C AUCHY problem for Laplace equation is a well-known inverse problem. Solutions to this problem are highly sensitive to variations in Cauchy data and small measurement noise can totally destroy the solution [1]. This mathematical problem is used to model a diverse range of physical phenomena. One of the applications is to recover actual heart electric potential using electrocardiogram (ECG) data on the body torso [2]. Other applications include nondestructive testing, electrical potential problem, corrosion problem, steady state diffusion phenomena [3]-[5] and some geophysical applications [6].

There have been many efforts going on over the past few decades to find stable solutions of this model problem for noisy data. The solution fails to fulfill Hadamard's stability criteria for non-smooth data [7] and some a priori assumption on the solution is a requirement to force the solution to continuously follow the data. This inverse problem was first introduced by Hadamard in 1902 [7] and since then there have been various proposed solution techniques. In general these techniques can be classified into two broad categories, namely, optimization-based techniques and observer-based techniques. Optimization-based techniques include various regularization methods developed to tackle this challenging problem. Just to name a few, a Tikhonov kind of regularization is introduced in [6], [8]. In [9] a quasireversibility method is introduced. An energy regularization

M.U. Majeed is a PhD candidate in Computer, Electrical and Mathematical Sciences and Engineering CEMSE, King Abdullah University of Science and Technology KAUST, K.S.A. e-mail: muhammadusman.majeed@kaust.edu.sa

T.M. Laleg-Kirati ${ }^{\star}$ is an assistant professor in CEMSE, KAUST, K.S.A. email: taousmeriem.laleg@kaust.edu.sa method is introduced in [10]. Regularization methods in general affect the solution depending on the choice of a particular regularization parameter and it also increase the computation cost. On the other hand, there are some methods based on state observer that transform the elliptic problem into a hyperbolic one by introducing an additional time variable like [11], [12]. Then a Luenberger type of observer [13] is developed for the transformed hyperbolic problem. First major draw-back of this kind of approach is the computational cost of introducing a new variable, that is, a two dimensional problem is transformed into a three dimensional problem. Second the performance of these techniques for noisy data is not well studied. Another kind of observer based technique to solve Cauchy problem for Laplace equation for only smooth Cauchy data is introduced in [14], where the idea is to use one of the space variables as a time-like variable to reduce numerical computation cost.

This paper is an extension of the observer based technique presented in [14] to non-smooth and noisy data. Cauchy problem for the Laplace equation is presented as a first order system and an optimal mean square error (MSE) minimizer algorithm is developed to solve the problem. Algorithm developed in this paper can be seen as a Kalman filter-like optimal estimator [15] with space variable as a time-like variable. In following sections the boundary value problem is presented as a first order system in space. Next solving algebraic Riccati equations optimal estimator gain is calculated. Numerically algorithm is implemented using finite difference discretization schemes. The technique presented in the given form is implemented by assuming fictitious points on the unknown solution boundary. Finally numerical results are presented for different levels of noise in Cauchy data.

\section{Problem Formulation}

Let $\Omega$ be a bounded annulus domain in $\mathbb{R}^{2}$ with two boundaries $\Gamma_{\text {in }}$ and $\Gamma_{\text {out }}$ as shown in figure 1. Cauchy problem for Laplace equation in polar co-ordinates is given as,

$$
\begin{aligned}
& \text { Find } u(r, \theta) \in \Gamma_{i n}: \\
& \qquad \begin{cases}\triangle u=r^{2} \frac{\partial^{2} u}{\partial r^{2}}+r \frac{\partial u}{\partial r}+\frac{\partial^{2} u}{\partial \theta^{2}}=0 & \text { in } \Omega, \\
\frac{\partial u}{\partial r}=\tilde{g}(r, \theta) & \text { on } \Gamma_{\text {out }}, \\
u(r, \theta)=\tilde{f}(r, \theta) & \text { on } \Gamma_{\text {out }},\end{cases}
\end{aligned}
$$

where $\tilde{g}=g+\omega$ and $\tilde{f}=f+v$ represent perturbed Cauchy data with $\omega\left(\eta_{1}, \sigma_{1}\right)$ and $v\left(\eta_{2}, \sigma_{2}\right)$ as additive white Gaussian noise with mean values $\eta_{1}, \eta_{2}$ and variances $\sigma_{1}, \sigma_{2}$ 
respectively. In the following subsection, we propose to rewrite this ill-posed boundary value problem in state-spacelike representation using one of the space variables as a time-like variable.

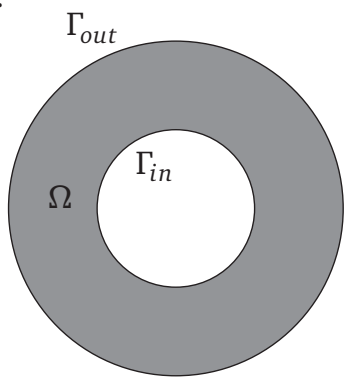

Figure 1. Annulus domain $\Omega$ with inner boundary $\Gamma_{\text {in }}$ and outer boundary $\Gamma_{\text {out }}$.

Let us introduce two auxiliary variables $\xi_{1}(r, \theta)$ and $\xi_{2}(r, \theta)$ such that,

$$
\left\{\begin{array}{l}
\xi_{1}(r, \theta)=u(r, \theta), \\
\xi_{2}(r, \theta)=\frac{\partial u}{\partial \theta} .
\end{array}\right.
$$

Further re-write the Laplace equation as a first order state equation as,

$$
\dot{\xi}=\mathscr{A} \xi,
$$

with,

$$
\begin{aligned}
\xi(r, \theta) & =\left(\begin{array}{l}
\xi_{1}(r, \theta) \\
\xi_{2}(r, \theta)
\end{array}\right), \\
\mathscr{A} & =\left(\begin{array}{cc}
0 & 1 \\
-r^{2} \frac{\partial^{2}}{\partial r^{2}}-r \frac{\partial}{\partial r} & 0
\end{array}\right),
\end{aligned}
$$

where represents partial derivative with respect to $\theta$. Now problem (1) can be reformulated as,

Find $\xi_{1}(r, \theta) \in \Gamma_{i n}$ :

$$
\begin{cases}\dot{\xi}=\mathscr{A} \xi & \text { in } \Omega, \\ \frac{\partial \xi_{1}}{\partial r}=\tilde{g}(r, \theta) & \text { on } \Gamma_{\text {out }}, \\ \xi_{1}(r, \theta)=\tilde{f}(r, \theta) & \text { on } \Gamma_{\text {out }} .\end{cases}
$$

It is important to re-emphasis that nice analytical solution of the above problem exists for smooth Cauchy data $(f, g)$ only and arbitrarily small noise in Cauchy data can totally destroy the solution [7], [16]. In the following subsection a nice well-posed forward problem is presented. Solution of this well-posed forward problem will be used to test the accuray of optimal algorithm.

Let us define a well-posed stable boundary value problem for Laplace equation as follows,

$$
\begin{aligned}
& \text { Find }\left.\xi_{1}\right|_{\Gamma_{\text {out }}}= f(r, \theta): \\
& \qquad \begin{cases}\dot{\xi}=\mathscr{A} \xi & \text { in } \Omega, \\
\frac{\partial \xi_{1}}{\partial r}=g(r, \theta) & \text { on } \Gamma_{\text {out }}, \\
\xi_{1}(r, \theta)=h(r, \theta) & \text { on } \Gamma_{\text {in }},\end{cases}
\end{aligned}
$$

where $g$ and $h$ are noise free smooth boundary conditions. Solution of above forward problem provides Cauchy data $(f, g)$ on $\Gamma_{\text {out }}$. Now the inverse problem is to estimate $h(r, \theta)$ using noise-free $(f, g)$ and noisy $(\tilde{f}, \tilde{g})$ Cauchy data. In the following sections problem (6) is discretized using finite difference discretization and an optimal estimator is developed.

\section{DERIVATION OF OPTIMAL MSE MINIMIZER ALGORITHM}

Before proper algorithm formulation, let us discretize problem (6) using finite difference discretization.

\section{A. Numerical discretization}

Forward Euler-like approximation is used to discretize first derivative along $\theta$ as,

$$
\frac{\partial \xi}{\partial \theta} \approx \frac{1}{\Delta \theta}\left[\xi^{n+1}-\xi^{n}\right],
$$

where $n$ is discrete positive integer index for variable $\theta$ and $\triangle \theta$ is a small step size. State equation, after discretization in variable $\theta$, can be written as,

$$
\xi^{n+1}=(I+(\triangle \theta) \mathscr{A}) \xi^{n},
$$

here $I$ is $2 \times 2$ identity matrix. Second equation in (9) can be written as,

$$
\left(\xi_{2}\right)^{n+1}=\left(\xi_{2}\right)^{n}+\Delta \theta\left(-r^{2} \frac{\partial^{2}}{\partial r^{2}}-r \frac{\partial}{\partial r}\right)\left(\xi_{1}\right)^{n},
$$

Next to discretize in variable $r$, following second order accurate finite difference discretization approximations are used to discretize first and second order derivative in above equation.

$$
\begin{aligned}
\frac{\partial}{\partial r}\left(\xi_{1}\right)^{n} & \approx \frac{1}{2(\Delta r)}\left[\left(\xi_{1}\right)_{i+1}^{n}-\left(\xi_{1}\right)_{i-1}^{n}\right] \\
\frac{\partial^{2}}{\partial r^{2}}\left(\xi_{1}\right)^{n} & \approx \frac{1}{(\Delta r)^{2}}\left[\left(\xi_{1}\right)_{i+1}^{n}-2\left(\xi_{1}\right)_{i}^{n}+\left(\xi_{1}\right)_{i-1}^{n}\right]
\end{aligned}
$$

$i$ is the discrete index for variable $r, i=1$ on $\Gamma_{i n}$ and $(\Delta r)$ is a reasonably small step size along $r$. Now from (10),

$$
\begin{array}{r}
\frac{1}{\triangle \theta}\left[\left(\xi_{2}\right)_{i}^{n+1}-\left(\xi_{2}\right)_{i}^{n}\right]=\frac{-r_{i}^{2}}{(\triangle r)^{2}}\left[\left(\xi_{1}\right)_{i+1}^{n}-2\left(\xi_{1}\right)_{i}^{n}+\left(\xi_{1}\right)_{i-1}^{n}\right] \\
+\frac{-r_{i}}{2(\Delta r)}\left[\left(\xi_{1}\right)_{i+1}^{n}-\left(\xi_{1}\right)_{i-1}^{n}\right],
\end{array}
$$

rewriting above equation on inner boundary $\Gamma_{i n}$, that is, at $i=1$ gives,

$$
\begin{array}{r}
\frac{1}{\triangle \theta}\left[\left(\xi_{2}\right)_{1}^{n+1}-\left(\xi_{2}\right)_{1}^{n}\right]=\frac{-r_{1}^{2}}{(\Delta r)^{2}}\left[\left(\xi_{1}\right)_{2}^{n}-2\left(\xi_{1}\right)_{1}^{n}+\left(\xi_{1}\right)_{0}^{n}\right] \\
+\frac{-r_{1}}{2(\triangle r)}\left[\left(\xi_{1}\right)_{2}^{n}-\left(\xi_{1}\right)_{0}^{n}\right] .
\end{array}
$$

As state earlier, index $i=1$ represents point on $\Gamma_{i n}$ and thus $i=0$ represents fictitious points outside domain $\Omega$ and close 
to inner boundary $\Gamma_{i n}$, as shown in Figure 2. Taking out fictitious points $\left(\xi_{1}\right)_{0}^{n}$ from the above equation gives,

$$
\begin{array}{r}
\left(\xi_{1}\right)_{0}^{n}=\frac{1}{r_{1}^{2}-\frac{\Delta r}{2} r_{1}}\left[-\left(r_{1}^{2}+\frac{(\Delta r)}{2} r_{1}\right)\left(\xi_{1}\right)_{2}^{n}+2 r_{1}^{2}\left(\xi_{1}\right)_{1}^{n}\right] \\
-\frac{1}{r_{1}^{2}-\frac{\Delta r}{2} r_{1}}\left[\frac{(\Delta r)^{2}}{\triangle \theta}\left\{\left(\xi_{2}\right)_{1}^{n+1}-\left(\xi_{2}\right)_{1}^{n}\right\}\right] .
\end{array}
$$

It is important to re-emphasis here that our objective is to develop an optimal algorithm which runs iteratively using $\theta$ as a time-like variable. Right hand side of equation (10) only depends on variable $r$. Thus fictitious point $\left(\xi_{1}\right)_{0}^{n}$ for a particular $n$ can be found uniquely as it depends on $\left(\xi_{2}\right)_{1}^{n+1}$. The idea of assuming fictitious points on the inner boundary can be understood as an assumption that Laplace equation is satisfied on the inner boundary. That is, we are trying to solve a $2 \mathrm{D}$ problem line by line assuming that,

$$
\frac{\partial^{2} \xi_{1}}{\partial \theta^{2}}=-\left(r \frac{\partial \xi_{1}}{\partial r}+r^{2} \frac{\partial^{2} \xi_{1}}{\partial r^{2}}\right) \text { on } \Gamma_{i n},
$$

which is an applicable boundary condition on $\Gamma_{i n}$ in the current state space-like setup. At the start of the algorithm, $\left(\xi_{1}\right)_{1}^{n},\left(\xi_{1}\right)_{2}^{n},\left(\xi_{2}\right)_{1}^{n}$ and $\left(\xi_{2}\right)_{1}^{n+1}$ come from the initial guess over the domain $\Omega$. These fictitious points are used in optimal estimator equations to tackle the boundary condition on the inner boundary, whereas Neumann boundary condition $\tilde{g}$ is used to tackle the boundary condition on outer boundary.

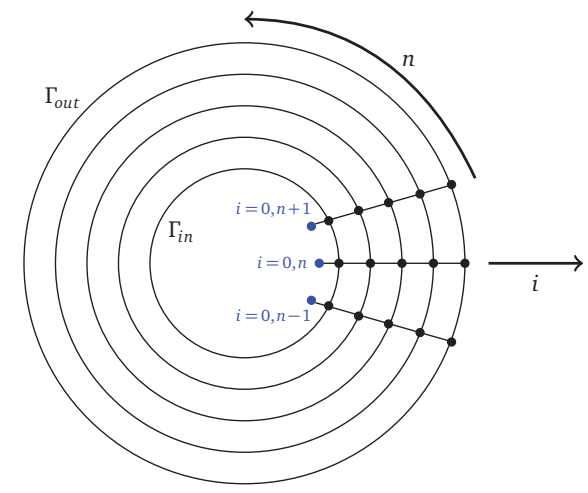

Figure 2. Fictitious points close to inner boundary $\Gamma_{i n}$, with index $i=0$.

\section{B. Optimal estimator derivation}

After full numerical discretization the state equation can be written as,

$$
\xi^{n+1}=(I+(\triangle \theta) A) \xi^{n} \quad \text { in } \Omega
$$

along with Neumann boundary condition $\frac{\partial \xi_{1}}{\partial r}=\tilde{g}(r, \theta)$ on $\Gamma_{\text {out }}$ and boundary condition given by equation (16) on $\Gamma_{i n}$. $A$ is the discrete version of $\mathscr{A}$ and $I$ is an identity matrix. Both $A$ and $I$ are square matrices of the same size with dimension depending on number of discretization points along $r$. Next Dirichlet boundary condition on $\Gamma_{\text {out }}$ can be written as a measurement or observation equation in discrete form as,

$$
y^{n}=C \xi^{n}+v^{n}=f^{n}+v^{n}
$$

where $C$ is a discrete observation matrix such that $C \xi^{n}=$ $\left.\left(\xi_{1}\right)^{n}\right|_{\Gamma_{\text {out }}}, f^{n}$ is the Dirichlet boundary condition at $\theta_{n}=$ $n \times \triangle \theta$ along with measurement noise $v^{n}$. An important condition to be satisfied for a system in state-space-like representation (17) and (18) is the system observability. Observability is equivalent to the existence of solution in linear dynamical theory [17], [18].

Definition 1. Observability is the measure of how well the internal states can be recovered from the measurements of output (observations). Let $\alpha$ be a positive integer constant, numerically system $\left(C, A^{\prime}\right)$, with $C$ as discrete observation matrix and $A^{\prime}$ as discrete state operator matrix of size $\alpha \times \alpha$, is called observable if,

$$
\operatorname{rank}(T)=\operatorname{rank}\left(\begin{array}{c}
C \\
C A^{\prime} \\
C A^{\prime 2} \\
\vdots \\
C A^{\prime \alpha-1}
\end{array}\right)=\alpha .
$$

In our case $A^{\prime}=(I+(\triangle \theta) A)$. Let the covariance matrices of process and measurement noise be stationary over $\theta$ and are given by,

$$
\begin{aligned}
Q & =E\left[\omega^{n}\left(\omega^{n}\right)^{T}\right], \\
R & =E\left[v^{n}\left(v^{n}\right)^{T}\right],
\end{aligned}
$$

where $Q$ is of the size of $(I+(\triangle \theta) A)$ and $R$ is a $1 \times 1$ matrix. Let $\hat{\xi}^{n}$ be the estimate of true state $\xi^{n}$. The difference of estimated and true state can be written as $e^{n}=\left(\xi^{n}-\hat{\xi}^{n}\right)$. It is important to consider the ability of the estimator to predict the states over a period of time-like variable $\theta$, hence a feasible metric is the expected value of positive definite error functional $\left(\xi^{n}-\hat{\xi}^{n}\right)^{2}$, given by,

$$
\epsilon(\theta)=E\left[\left(e^{n}\right)^{2}\right]=E\left[e^{n}\left(e^{n}\right)^{T}\right]=P^{n},
$$

where $P^{n}$ is the error covariance matrix at $\theta=n \times \Delta \theta$.

Proposition 1. Let pair $\left(C, A^{\prime}\right)$, with $A^{\prime}=(I+(\triangle \theta) A)$, be observable as given in (19) and $n$ be a discrete non-negative integer index for variable $\theta$. As $n \rightarrow \infty$ (that is, as $\theta \rightarrow \infty$, representing iterations over annulus domain), following two step algorithm minimizes the mean square error functional given in equation (22).

Prediction-step:

$$
\left\{\begin{array}{l}
\overline{\hat{\xi}}^{n}=A^{\prime} \hat{\xi}^{n-1} \quad \text { in } \Omega, \\
\bar{P}^{n}=A^{\prime} P^{n-1} A^{\prime T}+Q, \\
\overline{\hat{\xi}}^{n=0}=\bar{P}^{n=0}=0,
\end{array}\right.
$$

with Neumann boundary condition $\tilde{g}$ on $\Gamma_{\text {out }}$ and fictitious points close to $\Gamma_{\text {in }}$ 
given by equation (15), taking care of boundary conditions.

Correction-step:

$$
\left\{\begin{array}{l}
\hat{\xi}^{n}=\overline{\hat{\xi}}^{n}+K^{n}\left(y^{n}-C \overline{\hat{\xi}}^{n}\right), \\
K^{n}=\bar{P}^{n} C^{T}\left(C \bar{P}^{n} C^{T}+R\right)^{-1} \\
P^{n}=\left(I-K^{n} C\right) \bar{P}^{n}
\end{array}\right.
$$

where $\hat{\xi}^{n}$ is an estimate of $\xi^{n}, \overline{\hat{\xi}}^{n}$ is the prior-estimate of $\hat{\xi}^{n}$, $\bar{P}^{n}$ is the prior-estimate of state error covariance matrix $P^{n}$ given by equation (22). $Q$ and $R$ as given in equations (20) and (21) and $K^{n}$ is the gain matrix.

Remark 1. Before going to the proof, it is important to remark that the idea of iterations $(n \rightarrow \infty)$ replaces the asymptotic time in standard Kalman filter algorithm.

\section{Proof:}

Let us re-write equation (22) as,

$$
P^{n}=E\left[\left(\xi^{n}-\hat{\xi}^{n}\right)\left(\xi^{n}-\hat{\xi}^{n}\right)^{T}\right] .
$$

Let us assume the prior estimate of $\hat{\xi}^{n}$ is called $\overline{\hat{\xi}}^{n}$ and was obtained by the knowledge of the system (prediction). The correction equation can be written using the prior estimate with measurement data as follows,

$$
\hat{\xi}^{n}=\overline{\hat{\xi}}^{n}+K^{n}\left(y^{n}-C \overline{\hat{\xi}}^{n}\right) .
$$

$K^{n}$ is the gain matrix which will be derived in a moment. The term $\left(y^{n}-C \overline{\hat{\xi}}^{n}\right)$ is the innovation or measurement residual. Substituting equation (18) into (26),

$$
\hat{\xi}^{n}=\overline{\hat{\xi}}^{n}+K^{n}\left(C \xi^{n}+v^{n}-C \overline{\hat{\xi}}^{n}\right)
$$

Now substituting equation (27) into (25) gives,

$$
\begin{aligned}
P^{n}= & E\left[\left(\left(I-K^{n} C\right)\left(\xi^{n}-\overline{\hat{\xi}}^{n}\right)-K^{n} v^{n}\right)\right. \\
& \left.\left(\left(I-K^{n} C\right)\left(\xi^{n}-\overline{\hat{\xi}}^{n}\right)-K^{n} v^{n}\right)^{T}\right],
\end{aligned}
$$

taking the error of prior estimate $\left(\xi^{n}-\overline{\hat{\xi}}^{n}\right)$ as uncorrelated to measurement noise gives,

$$
\begin{array}{r}
P^{n}=\left(I-K^{n} C\right) E\left[\left(\xi^{n}-\overline{\hat{\xi}}^{n}\right)\left(\xi^{n}-\overline{\hat{\xi}}^{n}\right)^{T}\right]\left(I-K^{n} C\right)^{T} \\
+K^{n} E\left[v^{n}\left(v^{n}\right)^{T}\right]\left(K^{n}\right)^{T},
\end{array}
$$

now substituting equations (21) and (25) into above equation yields,

$$
P^{n}=\left(I-K^{n} C\right) \bar{P}^{n}\left(I-K^{n} C\right)^{T}+K^{n} R\left(K^{n}\right)^{T} .
$$

Above equation is the error covariance update equation and the diagonal contains mean squared error as shown,

$P^{n n}=\left(\begin{array}{ccc}E\left[e^{n-1}\left(e^{n-1}\right)^{T}\right] & E\left[e^{n}\left(e^{n-1}\right)^{T}\right] & E\left[e^{n+1}\left(e^{n-1}\right)^{T}\right] \\ E\left[e^{n-1}\left(e^{n}\right)^{T}\right] & E\left[e^{n}\left(e^{n}\right)^{T}\right] & E\left[e^{n+1}\left(e^{n}\right)^{T}\right] \\ E\left[e^{n-1}\left(e^{n+1}\right)^{T}\right] & E\left[e^{n}\left(e^{n+1}\right)^{T}\right] & E\left[e^{n+1}\left(e^{n+1}\right)^{T}\right]\end{array}\right)$, trace of above matrix is the mean squared error and it is to be minimized with respect to $K^{n}$. Re-writing equation (30) gives,

$$
P^{n}=\bar{P}^{n}-K^{n} C \bar{P}^{n}-\bar{P}^{n} C^{T}\left(K^{n}\right)^{T}+K^{n}\left(C \bar{P}^{n} C^{T}+R\right)\left(K^{n}\right)^{T},
$$

using the fact that trace of a matrix is equal to trace of its transpose, it can be seen that,

$$
T\left[P^{n}\right]=T\left[\bar{P}^{n}\right]-2 T\left[K^{n} C \bar{P}^{n}\right]+T\left[K^{n}\left(C \bar{P}^{n} C^{T}+R\right)\left(K^{n}\right)^{T}\right],
$$

where $T\left[P^{n}\right]$ is the trace of covariance matrix $P^{n}$. Differentiating with respect to $K^{n}$,

$$
\frac{d}{d K^{n}} T\left[P^{n}\right]=-2 C \bar{P}^{n}+2 K^{n}\left(C \bar{P}^{n} C^{T}+R\right),
$$

setting derivative equal to zero gives,

$$
\left(C \bar{P}^{n}\right)^{T}=K^{n}\left(C \bar{P}^{n} C^{T}+R\right),
$$

solving for $K^{n}$ gives,

$$
K^{n}=\bar{P}^{n} C^{T}\left(C \bar{P}^{n} C^{T}+R\right)^{-1} .
$$

Above equation is the optimal estimator gain equation which minimizes the mean square state estimation error. Substituting above equation into equation (30) yields,

$$
\begin{aligned}
P^{n} & =\bar{P}^{n}-\bar{P}^{n} C^{T}\left(C \bar{P}^{n} C^{T}+R\right)^{-1} C \bar{P}^{n} \\
& =\bar{P}^{n}-K^{n} C \bar{P}^{n} \\
& =\left(I-K^{n} C\right) \bar{P}^{n}
\end{aligned}
$$

which is the update equation for state error covariance matrix with optimal gain $K^{n}$. Equations (26), (36) and (37) develop an estimate of variable $x^{n}$. State prediction is achieved using state equation,

$$
\overline{\hat{\xi}}^{n+1}=A^{\prime} \hat{\xi}^{n},
$$

along with Neumann boundary condition $\tilde{g}$ on $\Gamma_{\text {out }}$ and equation (16) on $\Gamma_{i n}$. To complete the recursion it is important to find an equation which projects state error covariance matrix into next $\theta$-step, $\theta+1$. This is achieved by forming an expression for the prior error (prediction error).

$$
\begin{aligned}
\bar{e}^{n+1} & =\xi^{n+1}-\overline{\hat{\xi}}^{n+1}, \\
& =A^{\prime} e^{n}+\omega^{n} .
\end{aligned}
$$

Now extending equation (22) to $n+1$ gives,

$$
\begin{aligned}
\bar{P}^{n+1} & =E\left[\bar{e}^{n+1}\left(\bar{e}^{n+1}\right)^{T}\right], \\
& =E\left[\left(A^{\prime} e^{n}+\omega^{n}\right)\left(A^{\prime} e^{n}+\omega^{n}\right)^{T}\right],
\end{aligned}
$$

$e^{n}$ and $\omega^{n}$ are uncorrelated as $e^{n}$ is the error accumulated in previous $n$ steps and $\omega^{n}$ is the process error for $n$-th step. This implies,

$$
\begin{aligned}
\bar{P}^{n+1} & =E\left[A^{\prime} e^{n}\left(A^{\prime} e^{n}\right)^{T}\right]+E\left[\omega^{n}\left(\omega^{n}\right)^{T}\right], \\
& =A^{\prime} P^{n} A^{\prime T}+Q .
\end{aligned}
$$

This has completed the optimal estimator recursive loop. 


\section{Numerical Results}

For all numerical examples presented in this section an annular domain with $r \in[0.5,1]$ is considered. Number of states $\xi_{i}$ are chosen such that Kalman rank condition given in equation (19) is satisfied. Pair $(C, A)$ is observable for $N_{r} \leq 8$, where $N_{r}$ is the number of discretization points along $r$. The results presented in this section were obtained in just 3 to 4 iterations over the domain $\Omega$, running the algorithm for more iterations has no effect on results. Algorithm was tested for various test cases with smooth, non-smooth and noisy Cauchy data. Numerical results on a $\left(N_{r} \times N_{\theta}\right)=(8 \times 2000)$ grid are summarized in following two subsections, where $N_{\theta}$ is the number of discretization points along $\theta$.

\section{A. For smooth data}

Figure. 3 shows the solution obtained by solving wellposed problem (7) with $h=\sin (\theta)+\sin (3 \theta)$ and $g=0$. Now $\left.(f, g)\right|_{\Gamma_{\text {out }}}$ obtained by solving problem (7) is used as Cauchy data to find unknown $h$ on the inner boundary $\Gamma_{i n}$. Figure. 4 shows the solution obtained by the optimal iterative algorithm. Figure. 5 compares the true $h=\sin (\theta)+\sin (3 \theta)$ with the one obtained by the algorithm on $\Gamma_{i n}$. For smooth data case process and measurement noise co-variance was taken $\sigma_{1}=\sigma_{2} \approx 10^{-7} \sim 10^{-6}$, that is, putting more confidence into the process and also assuming very small error in data with $\eta_{1}=\eta_{2}=0$.

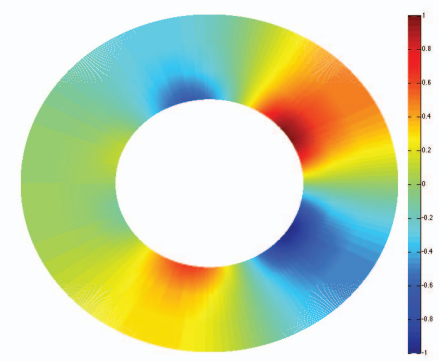

Figure 3. True solution or solution obtained by solving the problem (7) with $h=\sin (\theta)+\sin (3 \theta)$ and $g=0$ over $\Omega$.

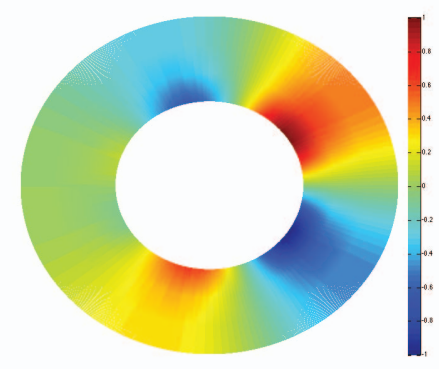

Figure 4. Solution obtained from optimal iterative algorithm over $\Omega$.

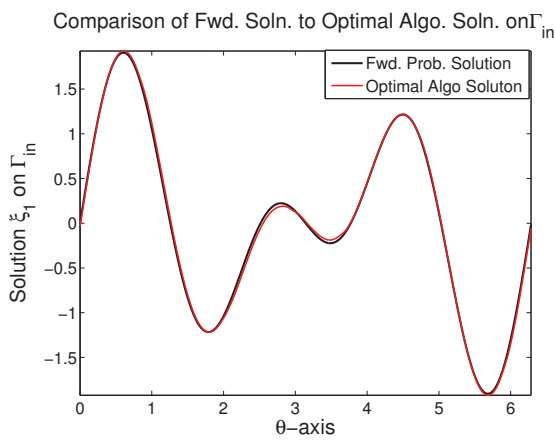

Figure 5. On $\Gamma_{i n}$, comparison of true boundary $h=\sin (\theta)+\sin (3 \theta)$ to the one recovered by optimal iterative algorithm using Cauchy data from $\Gamma_{\text {out }}$.

\section{B. For non-smooth data}

Figure. 6 shows the non-smooth pulse signal $h$ applied on $\Gamma_{i n}$. Further it shows the solution $f$ obtained by solving problem (7) with pulse-shaped signal $h$ at $\Gamma_{i n}$ along with Neumann zero boundary condition $(g=0)$ on $\Gamma_{\text {out }}$. Here problem (7) was solved using finite difference discretization. Figure. 7 compares true pulse-shaped signal and the one recovered by the algorithm on $\Gamma_{i n}$ using Cauchy data. Again as smooth data case, the process and measurement noise covariance was assumed to be very small $\sigma_{1}=\sigma_{2} \approx 10^{-7} \sim$ $10^{-6}$ and $\eta_{1}=\eta_{2}=0$. It can be seen that this algorithm is well-suited for edge detection of such a pulse-shaped signal.

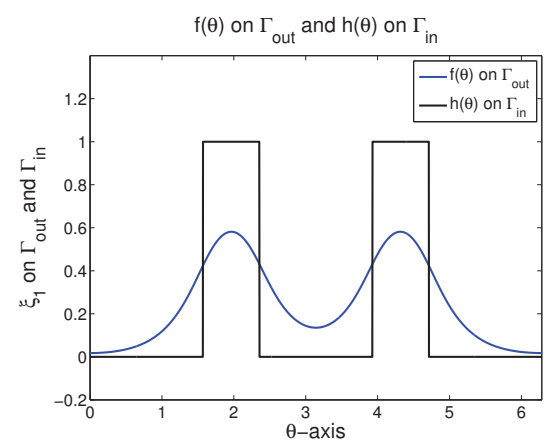

Figure 6. Solution of problem (7) $f(\theta)$ (in blue), by taking $h$ as a pulse signal and $g=0$.

\section{For noisy data}

In this section optimal iterative algorithm is studied for the case of noisy Cauchy data. First, problem (7) is solved using $h=\cos (\theta)$ on $\Gamma_{i n}$ and Neumann zero boundary condition $(g=0)$ on $\Gamma_{\text {out }}$. Solution of problem (7) provided $f$ on $\Gamma_{\text {out }}$. Next an additive white Gausian noise with $\eta_{2}=0$ and variance $\sigma_{2}=10^{-3}$ was added to $f$. This noisy $\tilde{f}$ along with Neumann zero ( $g=0$ ) was used as Cauchy data to solve the inverse problem. Process covariance $\sigma_{1}$ was assumed to be $\approx 10^{-4}$. Figure. 8 compares the true $h=\cos (\theta)$ with the one recovered by the algorithm using noisy Cauchy data. Figure. 9 shows the comparison of percentage relative error in Cauchy data on the outer boundary with 


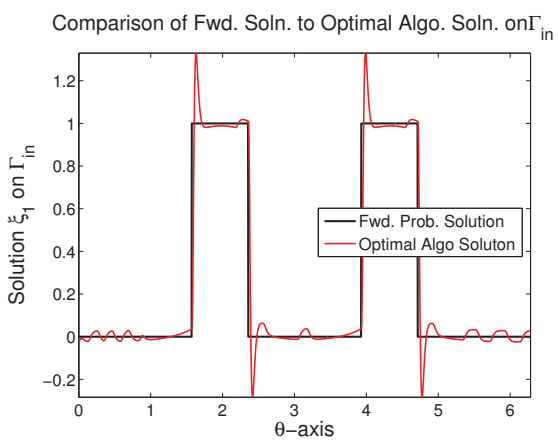

Figure 7. Comparison of true $h$ (a pulse signal) to the one recovered by optimal iterative algorithm using Cauchy data.

the percentage relative error in the recovered solution on the outer boundary (solution obtained using noisy Cauchy data). During all these measurement process noise variance $\sigma_{1}$ was taken as $10^{-4}$, whereas measurement noise variance $\sigma_{2}$ was adjusted according to the relative error in Cauchy data and $\eta_{1}=\eta_{2}=0$. Percentage relative error is given by,

$$
\% \text { Relative Error }=\frac{1}{\|h\|_{2}}\left\|h-h_{\text {recovered }}\right\|_{2} \times 100,
$$

where $\|$.$\| is the Eucledian 2-norm. It is obvious from$ Figure. 9 that error in the recovered solution is reduced with smaller and smaller error in Cauchy data. However for arbitrarily small noise in Cauchy data still there's around $2 \%$ relative error in solution, this might come from numerical discretization error.

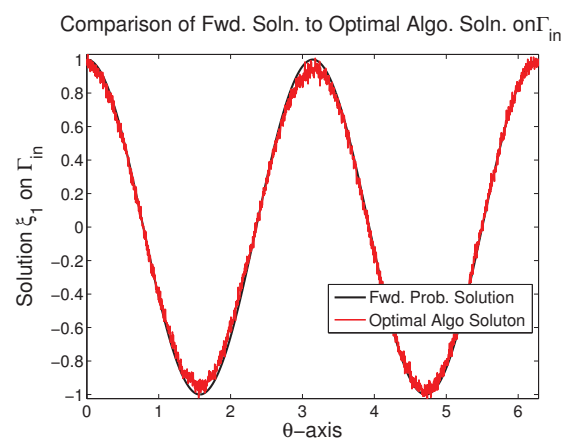

Figure 8. Comparison of true $h=\cos (\theta)$ to the one recovered by optimal iterative algorithm using noisy Cauchy data with measurement noise variance $\sigma_{2}=10^{-3}$.

\section{CONCLUSION}

An optimal iterative algorithm is presented to solve highly ill-posed Cauchy problem for Laplace equation. Using one of the space variables as a time-like variable an algorithm is developed to solve steady state boundary value problem. Stable and efficient numerical results for multiple test cases are presented. Algorithm developed in this paper happens to be robust to high noise in Cauchy data. Prospective research work includes the numerical discretization study and extension to more general shape of domains.

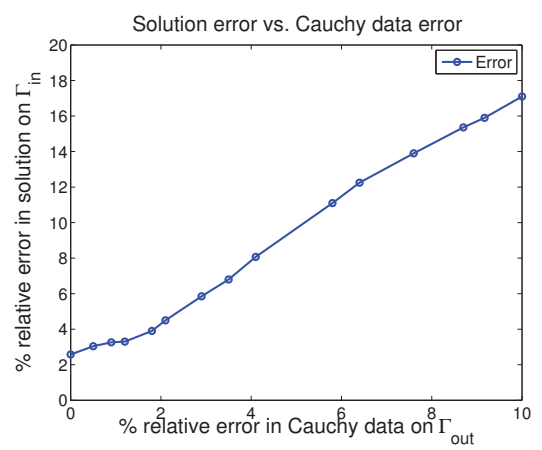

Figure 9. Percentage relative error in solution vs. percentage relative error in Cauchy data.

\section{ACKNOWLEDGMENT}

Research work presented in this manuscript is funded by King Abdullah University of Science and Technology (KAUST), Kingdom of Saudi Arabia.

\section{REFERENCES}

[1] V. Isakov, Inverse Problems for Partial Differential Equations, 2nd ed. Springer Berlin, 2006.

[2] R. Gulrajani, The forward and inverse problems of electrocardiography, IEEE Engineering in Medicine and Biology, pp.84-122, 1998.

[3] G. Alessandrini, Stable determination of a crack from boundary measurements, Proc. Roy. Soc. Edingburgh Sect. A 123 pp. 497-516, 1993.

[4] A.L. Bukhegeim, J. Cheng and M. Yamamoto, Conditional stability in an inverse problem of determining a non-smooth boundary, Journal of mathematical analysis and applications, vol. 242 pp. 57-74, 2000.

[5] J. Cheng and M. Yamamoto, Local stability of a linearized inverse problem in detecting steel reinforcement bars, Proc. of Int. Conf. on Inverse Problems and Applications, Quezon City, 1998.

[6] A.N. Tikhonov and V.Y. Arsenin Solutions of ill-posed problems, Winston and Sons, Washington, 1977.

[7] H.S. Hadamard, Sur les problemes aux drives partielles et leur signification physique, Princeton University Bulletin, pp. 49-52, 1902.

[8] D.D. Ang, N.H. Nghia and N.C. Tam, Regularized solution of Cauchy problem for Laplace equation in an irregular layer: a three dimensional model, Acta Math. pp. 65-74, Vietnam, 1998.

[9] M.V. Klibanov and F. Santosa, A computational quasi-reversibility method for Cauchy problem for Laplace equation, SIAM J. Appl. Math. pp. 1653-1675, 1999.

[10] H. Han and L. Ling and T. Takeuchi An Energy Regularization for Cauchy Problems for Laplace Equation in Annulus Domain, Commun. Comput. Phys. vol. 9, no. 4, pp. 878-896, 2011.

[11] M. Tadi, 2-D Inverse Heat Conduction Based on Observer Design, International Journal of Computational and Applied Mathematics ISSN 1819-499 vol. 7, no. 2, pp. 133-152, 2012.

[12] K. Berrier, D. Sorensen and D. Khoury, Solving the inverse problems for electrocardiography using Duncan and Horn formulation of the Kalman filter, IEEE Transactions on Biomedical Engineering, vol. 51(3), pp. 507-515, 2004.

[13] D. Luenberger, An introduction to observers, IEEE Trans. Aut Control, vol. 16, pp. 596-602, 1971.

[14] M.U. Majeed, C. Zayane-Aissa and T.M. Laleg-Kirati, Cauchy problem for Laplace equation: An Observer based Approach, The 3rd International Conference on Systems and Control, Algeria, 2013.

[15] R.E. Kalman, A New Approach to Linear Filtering and Prediction Problems, Journal of Basic Engineering 82(1), pp. 35-45, 1960.

[16] F. Berntsson and L. Elden, Numerical Solution of a Cauchy problem for Laplace equation, Inverse Problem, pp. 839-853, 2001.

[17] R.W. Brockett, Finite Dimensional Linear Systems, John Wiley \& Sons. ISBN 978-0-471-10585-5, 1970.

[18] D. Angeli and E.D. Sontag Forward completeness, unboundedness observability, and their Lyapunov characterization, Systems \& Control Letters (38), pp. 209-217, 1999. 